Acta Universitatis Wratislaviensis No 3742

Studia Linguistica XXXV Wrocław 2016

DOI: $10.19195 / 0137-1169.35 .2$

IWONA BARTOSZEWICZ

Uniwersytet Wrocławski, Polen

\title{
Linguistik und Rhetorik. Berührungspunkte
}

\section{Der linguistische Kompetenzbereich nach Ferdinand de Saussure}

Das Streben nach Interdisziplinarität, das Überschreiten von Grenzen zwischen nicht selten voneinander weit entfernten wissenschaftlichen Disziplinen sowie einen gewissen theoretischen und methodologischen Synkretismus könnte man vielleicht als immer häufiger auftauchende Merkmale zahlreicher, nach der sog. pragmatischen Wende entstandener linguistischer Veröffentlichungen betrachten. Wenn man das gleichzeitig als eine Art Tendenz identifiziert, dann könnte der Betrachter dieser Gegebenheiten vielleicht den Eindruck bekommen, dass er es in bestimmten Bereichen der Sprachwissenschaft mit einer zunehmenden Verschiebung der Schwerpunkte zu tun hat.

Es muss gleichzeitig aber deutlich zum Ausdruck gebracht werden, dass es sich in diesem Fall meist um Publikationen solcher Art handelt, die versuchen, sich durch das bloße Erwähnen der sich außerhalb vom linguistischen Stammbereich befindenden Begriffe oder Forschungsmethoden als interdisziplinär zu verstehen und zu definieren. Unter dem hier genannten Stammbereich verstehen wir die strukturalistisch orientierte Linguistik nach Ferdinand de Saussure.

De Saussure und seine Nachfolger haben für viele Jahrzehnte die Positionierung und die Entwicklungsrichtung der Sprachforschung determiniert. In dem, aus dieser Perspektive betrachtet, fundamentalen Werk „Grundlagen der allgemeinen Sprachwissenschaft" (de Saussure 1967) des berühmten Schweizers, bestimmt der Autor den Gegenstand und die Aufgaben der Sprachwissenschaft, indem er schreibt: „Den Gegenstand der Sprachwissenschaft bilden zunächst alle Betätigungen des menschlichen Sprachvermögens [...] 
Die Aufgabe der Sprachwissenschaft ist also:

a) die Beschreibung und Geschichte vor allen erreichbaren Sprachen zu liefern, was darauf hinausläuft, die Geschichte der Sprachfamilien zu schaffen und nach Möglichkeit die Grundsprachen jeder Sprache zu rekonstruieren;

b) die Kräfte aufzusuchen, die jederzeit und überall in allen Sprachen wirksam sind, und die allgemeinen Gesetze abzuleiten, auf welche man alle speziellen Erscheinungen der Geschichte zurückführen kann;

c) sich abzugrenzen und sich selbst zu definieren“".

Das im letzten Punkt erwähnte „sich [A]bgrenzen und sich selbst [D]efinieren“ versteht de Saussure - was sich aus der Lektüre der weiteren Absätze der hier angesprochenen Monographie ergibt - als wünschenswerte Maßnahme gegen das zu weite Bestimmen des Forschungsfeldes dieser Disziplin, denn „die Grenzen, welche die Wissenschaften scheiden, sind nicht immer deutlich erkennbar" (de Saussure 1967:7). Aus diesem Grund seien alle Relationen der Sprachwissenschaft zur Sozialpsychologie, Anthropologie, Ethnographie und sogar zur Philologie offensichtlich, weil die Forscher in diesen, aber auch in anderen Fällen mit Texten umgehen (de Saussure 1967:8). Er ist aber der Meinung, dass der Interessenbereich der Sprachwissenschaft ganz genau bestimmt werden soll, wobei die Sprache vom Sprechen deutlich abgegrenzt werden muss, die beiden Bereiche aber selbstverständlich einander bedingen (de Saussure 1967:9-27).

Bei der Analyse des Textes von de Saussure zeichnet sich eine Tendenz $\mathrm{ab}$, den linguistischen Bereich exakt dadurch zu bestimmen, dass man einen sprachzentrierten Standpunkt präsentiert. Es kommt deutlich im V. Kapitel (de Saussure 1967:24-27) zum Ausdruck, in dem der Autor durch das Definieren des inneren und des äußeren Bezirks der Sprachwissenschaft einen für eine längere Zeit erfolgreichen und aus der Perspektive der Entwicklung der Sprachwissenschaft schwerwiegenden Versuch unternommen hat, das nicht Zutreffende zu streichen. Und das, was bleibt, ist eben die sog. innere Sprachwissenschaft, was das interpretatorische (und im nächsten Schritt sicher auch im gewissen Sinn das methodologische) Belieben reduzieren sollte. ${ }^{1}$ Der Autor definiert die Linguistik durch ihren Forschungsbereich, den die Sprache selbst („eine Sache für sich“ [de Saussure 1967:17]) bildet, die ein System ist, „das nur seine eigene Ordnung zulässt" (de Saussure 1967:27). Die in dieser Definition auffallende Personifika-

\footnotetext{
${ }^{1}$ Der Forscher scheint an die Festigkeit und Selbstdisziplin von diesem „Teil der menschlichen Rede“ zu glauben, der „unabhängig vom Einzelnen“ ist, „welcher für sich allein sie weder schaffen noch umgestalten kann; sie besteht nur kraft einer Art Kontrakt zwischen den Gliedern der Sprachgemeinschaft" (de Saussure 1967:17). Der Mensch wird hier zum Sprachnutzer reduziert, der aber im Sprechen, begriffen als „ein individueller Akt des Willens und der Intelligenz“, vom grammatikalischen System, ,das virtuell in jedem Gehirn existiert, oder in den Gehirnen einer Gesamtheit von Individuen“ bleibt, Gebrauch macht (de Saussure 1967:16).
} 
tion der Sprache ${ }^{2}$ ergibt sich aus der Annahme des Autors der „Grundfragen der allgemeinen Sprachwissenschaft", dass es zu empfehlen ist, „die Sprache vom Sprechen“ und daher auch „das Soziale vom Individuellen“, „,das Wesentliche vom Akzessorischen und mehr oder weniger Zufälligen“ (de Saussure 1967:16) zu scheiden.

\section{Das Problem des Ordnungsprinzips in der Sprachwissenschaft}

Ohne sich in das Thema weiter zu vertiefen, kann man nach Wildgen (2010) feststellen, dass es sich im Falle der durch de Saussure begründeten, wie übrigens im Falle aller Theorien, die die relativ junge wissenschaftliche Disziplin ${ }^{3}$ fundieren, um Metaphern handelt, die die Rolle des Definiens, bzw. des Explikans übernehmen. Solche metaphorischen Ausdrücke wie Produkt und Organismus ${ }^{4}$ (Wildgen 2010:7-10) sind nicht nur als Versuche zu verstehen, das Unverständliche verständlich zu machen bzw. den Eindruck zu vermitteln, dass das oben Genannte unter bestimmten Umständen in Frage kommt, sondern es bedeutet darüber hinaus, dass wir es bei der Suche nach den Grenzen der Sprache und der Sprachwissenschaft immer noch mit offenen Fragen zu tun haben. Es handelt sich dabei um Fragen von grundlegender Bedeutung.

Das Bestehen dieser offenen Fragen hat, wenn wir die Hypothese des vorhandenen Kernbereichs bzw. Systems einer natürlichen Sprache akzeptieren, sicherlich einen prozedural-interpretatorischen, metasprachlichen Charakter. Die Er-

2 Ein paar Zeilen weiter stellt der Autor das Folgende fest: „Die Sprache ist nicht eine Funktion der sprechenden Person; sie ist das Produkt, welches das Individuum in passiver Weise einregistriert; sie setzt niemals eine vorherige Überlegung voraus, und die Reflexion ist dabei nur beteiligt, sofern sie die Einordnung und Zuordnung betätigt [...]“" (de Saussure 1967:16).

${ }^{3}$ Als souveräne, wissenschaftliche Disziplin, die sich um das Bestimmen linguistischer Forschungsfelder bemüht und auf das Erarbeiten einer methodologischen und interpretatorischen Basis ausgerichtet ist, konnte die Sprachwissenschaft erst Anfang des 20. Jahrhunderts bestimmt und im Kontext anderer Wissenschaften verortet werden. Es ist dabei zu betonen, dass es sich hier um einen ziemlich komplizierten und auf Kompromissen beruhenden Prozess des sich Herauskristallisierens eines linguistischen Kerngebietes handelt.

${ }^{4}$ Diese durch die Evolutionstheorie inspirierte Vorstellung, dass wir es im Fall der natürlichen Sprachen mit Organismen zu tun hätten, mit allen Folgen, die sich aus solch einer Annahme ergeben, hat für viele Jahrzehnte die Richtung der Entwicklung der modernen, postromantischen Sprachwissenschaft determiniert. Leitgedanke war, dass das Subjektive an der Interpretation der sprachlichen Phänomene auszuschließen sei, hat man die Betrachtung der Sprache als eines der Objekte der materiellen Welt postuliert. Die philologische Betrachtungsperspektive versuchte man, zum Teil mit Erfolg, durch die naturwissenschaftliche zu ersetzen. Dieser Art von Umdenken in der Linguistik führte zum Entwurf verschiedener, im gewissen Sinn kompatibler Sprachmodelle. Es steht außer Frage, dass man dadurch im methodologischen Bereich neue Lösungen vorschlagen konnte. 
gebnisse der Bemühungen der strukturorientierten Linguistik, das Sprachsystem zu definieren, scheinen im Allgemeinen nicht zufriedenstellend zu sein. ${ }^{5}$ Diese Fragen betreffen im methodologischen Bereich zum Beispiel den linguistischen Modus procedendi, der jedes Mal durch eine konkrete Theorie und in einem gewissen Sinn durch die mit dem Sprachbereich inkompatible Tradition, die auf andere Bereiche der Wissenschaft verweist, beeinflusst wird.

In diesem Kontext sollte das oben bereits angedeutete Problem der zum Darstellen unterschiedlicher Aspekte des Sprachsystems gebrauchten Metasprache kurz angesprochen werden.

\section{Die Linguistik als Nehmerdisziplin}

Josef Kopperschmidt schreibt in einer für das Wiederbeleben der rhetorischen Forschung und Lehre im deutschsprachigen Raum wichtigen Veröffentlichungen, dass der homo rhetoricus sich in einer Interaktion strategisch verhalten muss, um die negative Wirkung der unerwünschten Folgen von getroffenen Entscheidungen auszuschließen. Das bedeutet, dass die Meinungen derer nicht ignoriert werden sollten, die das Ergebnis einer Verhandlung mitbestimmen, weshalb man sich dem Gegenüber ,knechtisch“ unterwerfen müsse, wenn man „sich durch Reden behaupten will“" (Kopperschmidt 1999:13). Nach Friedrich Nietzsche, der meinte: „die Sprache ist Rhetorik, denn sie will nur eine doxa, keine episteme übertragen"66, betreffen diese Worte uns alle, weil wir unsere Meinungen und nicht das objektiv bestehende Wissen verbal geltend machen. Das trifft nach Nietzsche und Schoppenhauer ${ }^{7}$ insbesondere auf den Bereich des Wissenstransfers zu.

\footnotetext{
5 Dieter Wunderlich (1974) beschäftigt sich in Kapitel 9.11 seiner Monographie mit den Selektionsbeschränkungen, die zu beachten sind, wenn der Sprachnutzer (es ist hier die natürliche Sprache gemeint) dem Fehler „der möglichen Vagheit von Ausdrücken“ bzw. der möglichen „Sinnlosigkeit von Ausdrücken“ (1974:271) entkommen will. Zu diesem Zweck sind die Präsuppositionen von Ausdrücken auf ihre Falschheit oder ihre Sinnlosigkeit hin zu überprüfen. Das ermöglicht, die folgenden, grammatisch korrekten Sätze als sinnlos und daher als nicht akzeptabel einzustufen: „Die Semantiktheorie schnarcht. Am Morgen zwitschern die Schafe. Frege hat sich für die Transformationsgrammatik nicht interessiert. Willy Brandt hat eingesehen, dass er aus der CSU austreten sollte. Kuno hat die eigene Witwe geheiratet" (Wunderlich 1974:271).

${ }^{6}$ Vgl. Nietzsche (1995), nach Vetter/Heinrich (1999:13).

7 Schopenhauer schreibt (originale Ortographie): „Also die objektive Wahrheit eines Satzes und die Gültigkeit desselben in der Approbation der Streiter und Hörer sind zweierlei. (Auf letztere ist die Dialektik gerichtet.) Woher kommt das? - Von der natürlichen Schlechtigkeit des menschlichen Geschlechts. [...] Hienach hätte nun zwar bloß Jeder sich zu bemühen nicht anders als richtig zu urtheilen: wozu er erst denken und nachher sprechen müßte. Aber zur angeborenen Eitelkeit gesellt sich bei den Meisten Geschwätzigkeit und angeborene Unredlichkeit“" (1983-1990, http://www. rhetorik-netz.de/rhetorik/schopenh.htm\#Zur4. Zugriff am 08.06.2014).
} 
Die Linguistik als eine relativ junge wissenschaftliche Disziplin ${ }^{8}$, die sich ununterbrochen um ihre terminologisch-methodologische Souveränität bemüht, kann, was ihre Bereitschaft, von in anderen Disziplinen gängigen Lösungswegen Gebrauch zu machen, angeht, als Nehmerdisziplin ${ }^{9}$ bezeichnet werden. Es sind hier nicht unbedingt nur diejenigen Felder gemeint, die man für gewöhnlich mit den Geisteswissenschaften in Verbindung bringt. Die Suche nach der methodologischen Identität ist in diesem Fall zum Problem von besonderer Bedeutung geworden, denn die bisher getroffenen Entscheidungen scheinen keine auf die Dauer zufriedenstellenden Ergebnisse gebracht zu haben. Es gibt jedoch sicher mindestens folgende Gründe, die dafür zu sprechen scheinen.

- Probleme mit der Bestimmung des Bezugsbereichs: Wenn wir uns in unseren Überlegungen lediglich auf Interpretationsmöglichkeiten des Begriffs Sprache im Sinne der Theorie von de Saussure verlassen, laufen wir Gefahr, das, was am analysierten Gegenstand der genauen Analyse unterzogen werden soll, nur in beschränktem Maße zu erfassen. Ist es vielleicht immer noch der Bereich einer natürlichen Sprache (langue) oder sind wir vielleicht schon ein paar Schritte weiter gegangen und erfahren etwas über die Sprache überhaupt (language)?

- Probleme mit der Bestimmung des Forschungsinstrumentariums: Bei den in der strukturorientierten Linguistik so zahlreichen, um der Exaktheit willen formulierten Theorien und tradierten Verfahrensmodi, die nicht selten untereinander inkompatibel sind, bekommen wir den Eindruck, dass wir es mit verschieden Narrationen zu tun haben, die das gleiche Objekt oft unterschiedlich erfassen.

${ }^{8}$ Einige der heute bekannten sprachwissenschaftlichen Teildisziplinen wie Lexikographie, Lexikologie, Etymologie, Morphologie, Satzglieder- und Phrasenforschung, bestimmte Fragen der Stilistik, im gewissen Sinn die Textforschung und vor allen Dingen die Onomastik haben sich primär selbstständig oder im Rahmen anderer wissenschaftlicher Kontexte entwickelt (Philosophie, Logik, Rhetorik, Philologie usw.). Ohne Zweifel ist hier die allgemeine Namenforschung zu nennen, die schon Vorsokratiker als den Weg zur Entdeckung der Rolle des Namens, als Mittel zum Erkennen der Wahrheit des Seienden betrachtet haben. Zum zentralen Problem im Rahmen der so orientierten philosophischen Reflexion wurde die „Zuverlässigkeit des Wortes und des Benennens“ (Hennigfeld 1994:4).

${ }^{9}$ Wenn wir geneigt wären, die Sprachwissenschaft und ihre Entwicklung sowie ihre aus der hier vorgeschlagenen Perspektive dargestellte Situierung unter anderen wissenschaftlichen Disziplinen zu akzeptieren, sollten wir beachten, dass z.B. Philosophie, Logik, Biologie, Mathematik, Chemie, Kybernetik, Semiotik, Psychologie, Soziologie und selbstverständlich auch Rhetorik ihren Anteil am Sich-Ausformen der linguistischen Subdisziplinen, ihrer Theorie, Methodologie und Terminologie haben. Eine gewisse Heterogenität der Disziplin in den hier genannten Bereichen könnte aus wenigstens zwei Perspektiven betrachtet und ausgewertet werden. Optimistisch und positiv ist die Aufgeschlossenheit der Linguistik gegenüber anderen Forschungsbereichen, was eine Bereicherung der Sprachwissenschaft mit sich bringt bzw. vermuten lässt. Gleichzeitig aber könnte oder sogar sollte man sich der Grenzen und Folgen dieser Assimilationsprozesse bewusst sein. Bei dem anderen Gegenstand (die Sprache pur oder die Sprache und das Sprechen mit allen infrage kommenden Objekten) handelt es sich, wie es scheint, lediglich um bestimmte Anpassungsversuche der übernommenen Modelle, Termini und Methoden an die zu beschreibenden Gegenstände mit allen Einschränkungen, die sich aus den hier genannten Gründen ergeben. 
Und wenn es tatsächlich so ist, wofür vieles spricht, darf man dann vielleicht von verschiedenen oder wenigstens von vielen Standpunkten sprechen?

- Probleme mit der Metasprache: Es stellt sich nun die Frage, ob wir immer noch imstande sind, mit der in der Linguistik gebrauchten Metasprache produktiv umzugehen. Gibt es in der Sprachwissenschaft eine oder vielleicht mehrere Arten von Metasprache? Im Falle einer positiven Antwort müsste man entscheiden, ob die Nutzer dieser Metasprachen imstande sind, im Gespräch untereinander das Kooperationsprinzip von Grice einzuhalten. Sprechen wir immer noch über die natürliche Sprache als Gegenstand der wissenschaftlichen Reflexion oder vielleicht über die Möglichkeiten und Grenzen der Metasprache als stark formalisiertes und im Grunde genommen den Einflüssen einer natürlichen Sprache ausgesetztes Instrument der linguistischen Forschung? Als Nehmerdisziplin macht die Linguistik beispielsweise Gebrauch von der Fachterminologie der Logik, wobei aufgrund des unterschiedlichen Forschungsgenstands ${ }^{10}$ dieser Disziplinen die in der Linguistik gebrauchten Termini neu definiert werden müssen.

\section{Die Perspektive der Rhetorik}

Die Suche nach einer zufriedenstellenden Antwort auf die Frage, was die Wesensart von Sprache im Kontext anderer Elemente der materiellen Welt unter Einsatz von Methoden der Naturwissenschaften ist, scheint ihre Grenzen erreicht zu haben. Wenn man aber die Sprache nicht nur als souveränes Objekt der Kategorisierung, sondern in ihrer Relation zum Menschen analysiert ${ }^{11}$ oder sie auch durch ihre Rolle im Kontext der zwischenmenschlichen Beziehungen erklären will, so sind wir von der berühmten Definition der Sprache von Aristoteles nicht weit entfernt. Der Mensch als zoon politikon ist im Gegensatz zu den Tieren mit Sprache und Vernunft ausgestattet, um: „,das Nützliche und Schädliche mitzuteilen

\footnotetext{
${ }^{10}$ Im Fall der Linguistik sind das die (natürliche) Sprache und das Sprechen. Im Fall der Logik ist es das Denken, begriffen als Prozess mit einer konkreten Struktur und die Suche nach einer adäquaten, verbalen Darstellung dieses Prozesses. Sowohl im Fall der Logik als auch der Linguistik werden Fragen der Art gestellt, die darauf eingehen, wie der denkende und analysierende Mensch auf die Wirklichkeit Bezug nimmt.

11 Wenn wir vom Wesen der Sprache sprechen möchten, lässt sich die funktionale Komponente nicht ausschließen, was die mit der Prager Schule verbundenen Strukturalisten betont haben. Aber erst die Schriften der Forscher, die nach der sog. pragmatischen Wende die Rolle des Kommunikationsprozesses und der ihn konstruierenden Interaktanten aufgewertet haben, ließen auf eine neue Richtung der Entwicklung der Disziplin hoffen. Die Sprache, begriffen als Instrument, das Sprechen, begriffen als absichtlich ausgeführtes Handeln und der handelnde Mensch, der bei den bestehenden Ausdrucksmöglichkeiten über die Art und Weise der gewählten Mittel entscheidet, stehen im Brennpunkt des Interesses der pragmatischen Linguistik und der Rhetorik.
} 
und so auch das Gerechte und Ungerechte. Dies ist nämlich dem Menschen im Gegensatz zu den anderen Lebewesen eigentümlich, dass allein er die Wahrnehmung des Guten und Schlechten, des Gerechten und Ungerechten und anderer Moraldinge besitzt, und die Gemeinsamkeit in diesen Dingen schafft das Haus und den Staat" (Aristoteles 1973:18).

Die soziale und kulturbildende Rolle der Rede betont auch Ferdinand de Saussure (1967:8): ,[...] im Leben des einzelnen und der Gesellschaft gibt es nichts, was an Wirksamkeit und Wichtigkeit der Sprache gleichkommt".

In der antiken rhetorischen Fachliteratur wird ars rhetorica nicht als wissenschaftliche Disziplin identifiziert, denn sie ist als Kunst praxisorientiert. Gleichzeitig aber ist sie, worauf Quintilian in seinem für die Rhetorik und die Didaktik der Rhetorik grundlegenden Werk „Institutio oratoria“ an einigen Stellen hinweist, mehr als lediglich die Fertigkeit, zielorientiert zu sprechen: „Es geht mir um die Ausbildung des vollkommenen Redners, und das kann nur einer sein, der zugleich ein guter Mensch ist. Daher verlange ich von ihm nicht nur eine außergewöhnliche Redebefähigung, sondern auch alle charakterlichen Vorzüge. Ich erkläre mich nämlich nicht damit einverstanden, so wie einige das für richtig halten, die Grundsätze eines anständigen und ehrenhaften Lebens seien die Domäne der Philosophie. Denn der wahrhaft politisch gesinnte und zur Führung von Privat- und Staatsangelegenheiten befähigte Mann, der mit seinem Rat Städte leiten, ihnen durch seine Gesetzgebung eine feste Grundlage geben und durch sein richterliches Urteil Besserung verschaffen kann, dieser Mann ist niemand anderes als der Redner. Daher möchte ich, wenn ich auch zugebe, Gedanken aus philosophischen Schriften zu verwenden, doch bei der Anschauung bleiben, dass diese Dinge zu Recht und wahrheitsgemäß unsere Aufgabe sind und im eigentlichen Sinne zur Rhetorik gehören. Oder, wenn doch so häufig über Gerechtigkeit, Tapferkeit, Mäßigung und die übrigen Themen dieser Art gehandelt werden muss - und das geht so weit, dass es kaum einen Gerichtsfall gibt, bei dem keine solche Frage auftaucht - und wenn doch dies alles mit rednerischer Erfindungskunst und künstlerischem Ausdruck entwickelt werden muss, kann es da einen Zweifel geben, dass überall dort, wo Geisteskraft und Redefülle gefordert werden, das eigentliche Wirkungsgebiet des Redners liegt?"“ (Quintilianus 1974:9ff.).

Auch im 10. und 12. Buch dieses Werks, in denen Quintilian die Idee des ,perfekten Orators' als eines vir bonus dicendi peritus ${ }^{12}$ entwickelt, betont er die Rolle

12 Quintilian zeigt durch das in seinem Werk dargestellte komplexe Bildungsprojekt nicht nur die Möglichkeit, sondern sogar die Notwendigkeit, das durch Cato den Älteren mit der hier erwähnten Formel skizzierte Bild des perfekten Redners in der Wirklichkeit der römischen Republik ernst zu nehmen. Direkt formuliert es Quintilian im 12. Buch „Institutio oratoria“, indem er schreibt: „Für uns soll also der Redner, den wir heranbilden wollen, von der Art sein, wie ihn Marcus Cato definiert: >ein Ehrenmann, der reden kann $<$ - unbedingt jedoch das, was in Catos Definition am 
des agierenden Menschen (des Redners) im Kommunikationsprozess. Der Redner kann mittels seinem, durch ein umfassendes Wissen im langen und vielschichtigen Bildungsprozess (studium) erworbenes und sein natürliches (natura/ingenium), durch das Üben (exercitatio) und Nachahmen der besten Beispiele (imitatio) erfolgreich entwickeltes Talent den Verlauf der Interaktion nach entsprechenden Prinzipien gestalten. Das bedeutet, dass der angehende Redner in seinem Werdegang verschiedene Perspektiven beachten muss, aus welchen ein Kommunikationsereignis zu betrachten ist. Es sind, um nur einige davon zu nennen:

- die Perspektive der rhetorischen Theorie und Praxis ${ }^{13}$,

- die Perspektive des allgemeinen Wissens über die Dynamik des Kommunikationsprozesses,

- die Perspektive der konkreten Interaktion und der zu beachtenden Begleitumstände,

- die Perspektive des allgemeinen und/oder profilierten Sprach- und Weltwissens,

- die Perspektive der sozialen Rollen der Interaktanten und der gegenseitigen Wechselbeziehungen,

- die Perspektive der rhetorischen Wirkziele (Logos, Ethos und Pathos),

- die Perspektive der Autoritäten (Rhetorik, Politik, Kunst, Ethik, Philosophie, etc.),

- die Perspektive der sog. Hauptprinzipien der ars rhetorica ${ }^{14}$,

- weitere Perspektiven.

Anfang steht und auch seinem Wesen nach das Wichtigere und Größere ist: Ein Ehrenmann“ (1974:XII,1).

13 Die Ars retorica (gr. rhetoriké téchne ,Kunst der Rhetorik') wird in ihrer Geschichte unterschiedlich bewertet, nicht selten auch abgewertet (durch Platon verglichen im Dialog „Gorgias“ mit der Putzkunst, Kochkunst und identifiziert mit der Sophistik; durch Curtius für tot erklärt) oder aufgewertet, indem sie vom Aristoteles im Traktat „Rhetorik“ als Gegenstück zur Philosophie/Dialektik/Ethik dargestellt wird. Cicero versteht die Rhetorik als eine Disziplin, die dem Redner weitaus mehr abverlangt als andere Disziplinen: „Und nach meiner Ansicht wenigstens wird niemand ein in jeder Hinsicht vollkommener Redner sein können, wenn er sich nicht Kenntnisse von allen wichtigen Gegenständen und Wissenschaften angeeignet hat. Denn aus der Erkenntnis der Sachen muss die Rede erblühen und hervorströmen. Hat der Redner die Sachen nicht gründlich erfasst und erkannt, so ist sein Vortrag nur ein leeres und ich möchte sagen kindisches Gerede" (1873:I,VI,20). Aristoteles schreibt darüber im ersten Buch der Rhetorik, indem er den übergreifenden Charakter der Rhetorik darstellt. Thematisch fokussiert sie ihr Interesse nicht auf ihren eigenen Gegenstand (vgl. 1993).

${ }^{14}$ Korolko (1990:171) erwähnt dabei die ersten bekannten Theoretiker der rhetorischen Kunst, Korax und Theisjas, die vor über 2500 Jahren solche Parameter wie eikós (Wahrscheinlichkeit des Mitgeteilten), kairós (der richtige Moment) und prepon (Angemessenheit) erwähnt haben als diejenigen, die die Wirksamkeit der Rede determinieren. 


\section{Die Sprache im Kontext der rhetorischen Theorie und Praxis}

Die Rolle der Sprache ist in diesem breit dargestellten Kontext auf eine bestimmte Art und Weise zu verstehen. Als Instrument bzw. als eines der zum (persuasiven ${ }^{15}$ ) Kommunizieren durch den Menschen gebrauchten Instrumente determiniert die Sprache ihre Verwendungsart, was sich aus ihrer Eigenart ergibt. Das ist aber nicht so zu verstehen, dass die hier erwähnte Eigenart der Sprache dem Sprachbenutzer ${ }^{16}$ die Möglichkeit und Gelegenheit nimmt, den Sprachgebrauch in einem gewissen Sinn immer wieder neu zu gestalten. Der bewusste und gleichzeitig verantwortliche Umgang mit diesem Wissen charakterisiert die rhetorische Perspektive, aus welcher die Sprache als wichtiges Mittel der Persuasion betrachtet wird. Vom bewusst handelnden und gebildeten Menschen, der seine Rolle im sozialen Gefüge versteht, also vom Redner, erwarten wir, dass er sich selbst als Person erkennen lässt, die bereit ist, die gestörte oder bedrohte Harmonie der Welt, vor allen Dingen aber die Harmonie der sozialen Beziehungen, wiederherzustellen. ${ }^{17}$ Das bedeutet, was Cicero in den hier bereits angeführten $\mathrm{Zi}-$

15 Aus dem pragmalinguistischen Blickwinkel ist jedes Kommunizieren persuasiv. Davon zeugt das Bestehen der sog. Bedingungen für das Gelingen von Sprechakten (Sprechaktbedingungen) (Engel 2009:56-58), worunter die letzte uns besonders interessant ist. Sie nennt man ,Erfolgsbedingung", was bedeutet, dass erst eine aus der Perspektive des Senders erwünschte und zufriedenstellende Reaktion des Empfängers auf bestimmte Stimuli (in der rhetorischen Theorie und Praxis sind das nicht nur verbale Stimuli) das Gelingen des Sprechaktes verspricht.

${ }^{16}$ Mit dieser strukturalistisch orientierten Bezeichnung des sprechenden Menschen haben wir bestimmte Probleme, wie im ersten Teil dieses Beitrags aufgezeigt wurde.

17 Cicero schrieb in seinem Traktat „De republica“ vom Politiker als die in der Öffentlichkeit agierende Person, wobei die ethische Komponente der rhetorischen Kommunikation hervorgehoben wird. „Ich unterlasse die Aufzählung unendlich vieler Männer, von denen jeder zum Wohl dieses Staates beigetragen hat, und will hier an sie nicht weiter erinnern, da sie der Erinnerung unserer Zeit nicht unmittelbar nahe stehen, damit sich nicht jemand beklage, er oder einer der Seinen sei übergangen worden, und beschränke mich bloß auf die entschiedene Erklärung, dass in der menschlichen Natur eine solche innere Nötigung zur Tugend und ein solcher Drang, das Gemeinwohl zu verteidigen, liegt, dass dieser Trieb über alle Reize der Sinnenlust und behaglichen Muße die Oberhand gewonnen hat“ (Cicero, 1826:I,1.1). In einem anderen Traktat, in „De oratore“, definiert er die Aufgaben des Redners, indem er schreibt: „Und um auf die Beschäftigungen mit den geringfügigeren Künsten zu kommen, wenn die Frage den Tonkünstler, den Sprachforscher, den Dichter beträfe, so könnte ich auf ähnliche Weise erklären, was den Beruf eines jeden ausmache und was die erforderlichen Eigenschaften seien, auf die man sich bei jedem beschränken müsse. Ja selbst von dem Philosophen, der sich nach der Fülle seiner Weisheit allein im Besitz fast alles Wissens zu sein rühmt, findet doch eine gewisse Begriffsbestimmung statt, indem man demjenigen, welcher aller göttlichen und menschlichen Dinge Wesen, Beschaffenheit und Ursachen zu kennen, die ganze Sittenlehre zu wissen und auszuüben sich bemüht, diesen Namen erteilt. Was nun aber den Redner anlangt, der ja der Gegenstand unserer Untersuchung ist, so habe ich von ihm nicht dieselbe Vorstellung wie Crassus, der mir unter dem einen Namen und der einen Obliegenheit des Redners die gesamte Kenntnis aller Dinge und Wissenschaften zu begreifen schien; ich halte vielmehr den für 
taten deutlich zum Ausdruck bring, dass die Rhetorik, die Quintilian als Königin der Künste und Wissenschaften bezeichnet hat ${ }^{18}$, im Dienste einer übergreifenden Regel steht, die das Funktionieren der uns bekannten Welt und der Wesen, die diese Welt besiedeln, bestimmt und determiniert. Der Redner sollte sich dieser Regel und seiner Rolle in diesem Gefüge bewusst sein. Das Verankern der rhetorischen Verfahren im breiteren philosophischen und religiösen Kontext erscheint daher selbstverständlich. Es ist darüber hinaus so zu verstehen, dass man die Quellen der Ordnungsprinzipien bestimmter Bereiche der zu beschreibenden und zu analysierenden Wirklichkeit außerhalb dieser Bereiche suchen soll.

\section{Die Rhetorik als Geberdisziplin}

Angesichts dessen, was wir nach der Lektüre der antiken Theoretiker der Rhetorik über das Wesen und die Aufgaben dieser Kunst sagen können, ermöglicht dies einigermaßen klare Antworten auf die Frage nach dem Verhältnis zwischen Rhetorik und Linguistik:

- Die Rhetorik kann in ihrer Relation zur Sprachwissenschaft als Geberdisziplin bezeichnet werden.

- Der Kompetenzbereich der Rhetorik ist breiter als der Kompetenz- und Interessenbereich der Linguistik ${ }^{19}$ und der Philologie. In der Linguistik kann die pragmatische Wende ${ }^{20}$ als Anstoß betrachtet werden, die die Disziplin in die Nähe der rhetorischen Forschungstradition rückt.

- Die Rhetorik ist nicht nur Quelle vieler linguistischer Begriffe. Beispielsweise handelt es sich um Begriffe, die in der rhetorischen Theorie in den drei ersten Bearbeitungsphasen der Rede im Gebrauch sind: inventio, dispositio und

einen Redner, welcher in gerichtlichen und öffentlichen Verhandlungen Worte, die angenehm zu hören sind, und Gedanken, die Überzeugung einzuflößen geeignet sind, zu gebrauchen versteht. Einen solchen nenne ich einen Redner und wünsche, dass er außerdem auch Stimme, äußeren Vortrag und einen gewissen Witz besitze“" (Cicero, 1873:XLIX,212f.).

18 Das bedeutet, dass Quintilian gewissermaßen die Meinung von Aristoteles teilt, der am Anfang des ersten Buches seines Traktats „Rhetorik“ die ars rhetorica als Gegenstück zur Dialektik bezeichnet. Rhetorik und Dialektik hat Aristoteles als Methoden begriffen, die Angelegenheiten betreffen, die allen Menschen bekannt sind. Als Methoden sind sowohl die Rhetorik als auch die Dialektik Instrumente der Wahrheits- und Wahrscheinlichkeitsfindung.

19 Wissenschaftler, die sich mit der rhetorischen Problematik beschäftigen, vertreten verschiedene, vornehmlich philologische Disziplinen. Klassische Philologen, Mediävisten, Literaturforscher, Anglisten, Germanisten und Polonisten bestimmen die Entwicklungstendenzen der rhetorischen Forschung in Polen. Mirosław Korolko, Jakub Z. Lichański, Barbara Bogołębska, um nur einige Namen zu nennen, waren bzw. sind Mentoren einer sich in der letzten Zeit dynamisch entwickelnde Gruppe von Rhetorikforschern. Die Linguisten sind in diesem Kreis unterrepräsentiert.

20 Zeitlich wird die pragmatische Wende in der Linguistik an der Veröffentlichung der Monographie von John L. Austin „How to do things with words“ (1962) festgemacht. 
elocutio. ${ }^{21}$ Die linguistische Forschungs- und Beschreibungsmethodologie beruht im wesentlichen Teil auf der rhetorischen. ${ }^{22}$ Es handelt sich dabei selbstverständlich nicht um eine einfache Übertragung bestimmter Elemente, worauf wir bereits hingewiesen haben, vieles spricht jedoch dafür, dass die Rhetorik der Linguistik in dieser Hinsicht näher steht, als beispielsweise die Naturwissenschaften. Es gibt bestimmte Gründe, die sich dabei anführen lassen. Es sind zum Beispiel:

- der gemeinsame Interessenbereich - Sprache und (persuasives) Sprechen,

- in der Rhetorik und nach der pragmatischen Wende in der Linguistik: die Sprache begriffen als Instrument,

- sprechen begriffen als verbales, persuasives, interaktives Handeln,

- der Sprachnutzer - absichtlich und zielbewusst handelnder Interaktant (Proponent/Opponent),

- die Suche nach dem Ordnungsprinzip der Sprache und der Kommunikation (Rhetorik: Harmonie/Gott; Linguistik: eine für alle analytisch zugänglichen Objekte typische Selbstordnung/Konvention).

\section{Abschließende Bemerkung}

Die kanonisch begriffene Rhetorik und Linguistik scheinen die Grenzen ihrer Möglichkeiten in einem bestimmten Sinn erreicht zu haben. Die Bereitschaft, weitere, neue und gemeinsame Wege - einschließlich der Wege zueinander - zu finden, ist vorhanden. Davon zeugen zahlreiche und gelungene Versuche, vor der linguistischen Analyse neue Perspektiven zu eröffnen. ${ }^{23}$ Diese positive Einschätzung der bestehenden Möglichkeiten und der infrage kommenden Lösungen

21 Inventio, dispositio, elocutio, memoria und actio werden in der rhetorischen Fachliteratur auch als Sektoren der rhetorischen Kunst oder als Wirkziele der Rhetorik bezeichnet. Damit wird der ganze Produktions- und Darbietungsprozess der Rede erfasst. Der Rhetor definiert die Intention, die ihn zum rhetorischen Handeln bewegt und sucht nach entsprechenden Inhalten bzw. Methoden der Beweisführung, Affekterregung und der Charakterdarstellung, die nach seinem Wissen und seiner Erfahrung die Chancen auf den Erfolg im Streit optimieren sollten. Weiterhin muss der Redner entscheiden, wie er die gefundenen Inhalte hinsichtlich ihrer Wichtigkeit ordnen und welcher wirkungsvollen, sprachlichen Mittel er sich dabei bedienen sollte. Dann musste der Orator Methoden beherrschen, das Vorbereitete auswendig zu lernen und es zu memorisieren. Schließlich sollte nach Cicero die Rede würdevoll und elegant vorgetragen werden.

22 Vgl. dazu Bartoszewicz 2002, 2006, 2010.

23 Vgl. zwei Bände ( 3 u. 4) der polnischen, rhetorischen Fachzeitschrift „Forum Artis Rhetoricae“ (2012a, 2012b), die der gegenseitigen Wahrnehmung der Linguistik und Rhetorik und den persuasiven Texten als Objekt linguistischer Reflexion gewidmet sind. Sie sind als Versuch konzipiert worden, die bereits bestehende Bindung zwischen der ars rhetorica und der Linguistik sowie das gegenseitig Inspirierende darzustellen. 
scheinen auch die Rhetorikforscher zu teilen. Das lässt auf eine für beide Seiten günstige und gute Zukunft der beiden Disziplinen hoffen.

\section{Literatur}

ARISTOTELES, 1973, Politik. Übersetzt von Olof Gigon, München.

Aristoteles, 1993, Rhetorik. Aus dem Lateinischen von F.G. Sievke. 4. Auflage, München.

Austin John L., 1962, How to Do Things with Words, Cambridge.

BARTOSZEWICZ Iwona, 2006, Rhetorisch konnexe Texte, rhetorisch konnexe Diskurse, in: Grucza

F./Olpińska M./Schwenk J. (Hrsg.), Texte. Gegenstände germanistischer Forschung und Leh-

re. Materialien der Jahrestagung des Verbandes Polnischer Germanisten 12.-14. Mai 2006

Toruń, Warszawa, S. 71-84.

BARTOSZEWICZ Iwona, 2010, Rhetorische Komponenten der Textstruktur als translatorisches Prob-

lem, in: Małgorzewicz A. (Hrsg.), Translation: Theorie-Praxis-Didaktik, Wrocław/Dresden, S. $25-34$.

BARTOSZEWICZ Iwona (Hrsg.), 2012a, Forum Ars Rhetoricae 3: Linguistik und Rhetorik - gegenseitige Wahrnehmung, Warszawa.

BARTOSZEWICZ Iwona (Hrsg.), 2012b, Forum Ars Rhetoricae 4: Persuasive Texte als Objekt linguistischer Reflexion, Warszawa.

Cicero Marcus Tullius, 1826, De re publica libri, Gent.

Cicero Marcus Tullius, 1873, Vom Redner. De Oratore, Stuttgart.

CURTIUS Ernst Robert, ${ }^{11} 1993$, Europäische Literatur und Lateinisches Mittelalter, Tübingen/Basel.

ENGEL Ulrich, 2009, Deutsche Grammatik - Neubearbeitung, München.

HENNIGFELD Jochem, 1994, Geschichte der Sprachphilosophie. Antike und Mittelalter. Berlin/New York.

KINDT Walther, 2012, Linguistische Rhetorik, in: Forum Artis Rhetoricae 3(30), S. 31-46.

KopPERSCHMIDT Josef, 1999, Zur Modernität der Rhetorik. In: Mönnich A. (Hrsg.), Rhetorik zwischen Tradition und Innovation, München/Basel 1999, S. 10-17.

KorolKo Mirosław, 1990, Sztuka retoryki. Przewodnik encyklopedyczny, Warszawa.

NIETZSCHE Friedrich, 1995, Vorlesungsaufzeichnungen. WS 1871/72-WS 1874/75. Bearbeitet von

Fritz Bornmann und Mario Carpitella. KGW II, Berlin/New York.

Platon, 2013 ( $\left.{ }^{1} 1775\right)$, Gorgias, ein Gespräch von der Redekunst, Zürich.

QUINTILIANUS Marcus Fabius, 1974, Institutio oratoria, München.

SAUSSURE Ferdinand de, 1967, Grundfragen der allgemeinen Sprachwissenschaft. Herausgegeben

von Ch. Bally und A. Sechehaye unter Mitwirkung von H. Lommel. 2. Auflage mit neuem

Register und einem Nachwort von P. von Polenz, Berlin.

SCHOPENHAUER Arthur, 1983-1990, Eristische Dialektik oder Die Kunst, Recht zu behalten, Zürich.

VeTtER Helmut / HeINRICH Richard (Hrsg.), 1999, Die Wiederkehr der Rhetorik, Berlin.

WILDGEN Wolfgang, 2010, Die Sprachwissenschaft des 20. Jahrhunderts. Versuch einer Bilanz, Berlin/New York.

WUNDERLICH Dieter, 1974, Grundlagen der Linguistik, Hamburg. 